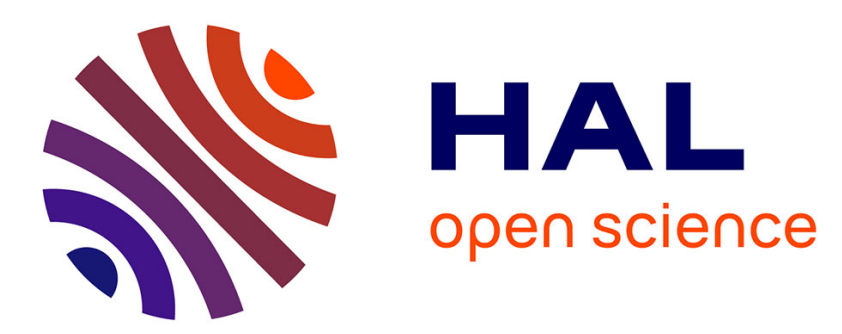

\title{
Unified Sensing Algorithm : a Smart Full Exploitation of Detection Methods
}

Kais Bouallegue, Jean-Yves Baudais, Matthieu Crussière

\section{To cite this version:}

Kais Bouallegue, Jean-Yves Baudais, Matthieu Crussière. Unified Sensing Algorithm: a Smart Full Exploitation of Detection Methods. International Conference on Wireless and Mobile Computing, Networking and Communications (WiMob 2019), Oct 2019, Barcelone, Spain. 10.1109/WiMOB.2019.8923570 . hal-02402500

\section{HAL Id: hal-02402500 https://hal.science/hal-02402500}

Submitted on 9 Oct 2020

HAL is a multi-disciplinary open access archive for the deposit and dissemination of scientific research documents, whether they are published or not. The documents may come from teaching and research institutions in France or abroad, or from public or private research centers.
L'archive ouverte pluridisciplinaire $\mathbf{H A L}$, est destinée au dépôt et à la diffusion de documents scientifiques de niveau recherche, publiés ou non, émanant des établissements d'enseignement et de recherche français ou étrangers, des laboratoires publics ou privés. 


\title{
Unified Sensing Algorithm : a Smart Full Exploitation of Detection Methods
}

\author{
Kaïs Bouallegue, Jean-Yves Baudais and Matthieu Crussière
}

\begin{abstract}
In this paper, we propose a new spectrum sensing (SS) algorithm called unified sensing algorithm (USA). This method exploits the results of different algorithms developed in the literature. The considered methods are especially those based on the covariance matrix of the received signal and those which further compute the eigenvalue decomposition of such matrix. More precisely, the USA algorithm implements some covariance matrix based methods, and in the case where the primary user is miss-detected, the eigenvalue decomposition is performed. This approach fully exploits all detection methods and avoids computational costs, as eigenvalue decomposition, when it is not necessary. Finally, the performance of the proposed method is provided and compared with existing state of the art algorithms. Simulation results show the performance improvement obtained with this new approach, namely the contribution in term of detection under very low signal-to-noise ratio (SNR).
\end{abstract}

Index Terms-Cognitive radio, spectrum sensing, covariance matrix, eigenvalue decomposition.

\section{INTRODUCTION}

In the next few years, the high demand for spectral resource will encourage the development of new key technology to overcome the scarcity of the spectrum and improve its exploitation. One of the main topic is the cognitive radio (CR) which proposes to define two kinds of users. The first one who has all the priorities on the spectral resource, called the primary user (PU), and the second one, called the secondary user (SU), who opportunistically exploits the free frequency bands [1]. In [2], the authors bring details about the cognition loop which is built upon the spectrum sensing (SS) algorithms. In order to not interfere with the PU, the $\mathrm{SU}$ has to be aware of its environment and the SS is one of the crucial step of efficient CR mechanism. For instance, in IEEE 802.22 wireless regional area networks (WRAN), unused frequency resources allocated for Digital TV (DTV) broadcasting are used in communication systems [3]. The requirement of this standard is to sense the PU at a SNR of at least $-21 \mathrm{~dB}$ with a probability of detection $\left(P_{d}\right)$ over 0.9 and a probability of false alarm $\left(P_{f a}\right)$ fixed at 0.1 [4]. All SS algorithms are based on two essential parameters, the first one, referred to as the statistical test, is extracted from the received signal of the focused frequency band and the second one, called the threshold, is fixed by the sensing conditions. In Fig. 1, we present one way of classification of SS algorithms. Cooperative SS algorithm is a good way to improve the performance of detection and to reduce the computational cost [5]. In order to optimize a cooperative dense network, some SS algorithms are developed grouping users in the same clusters [6]. In non-cooperative narrowband SS context, many algorithms are

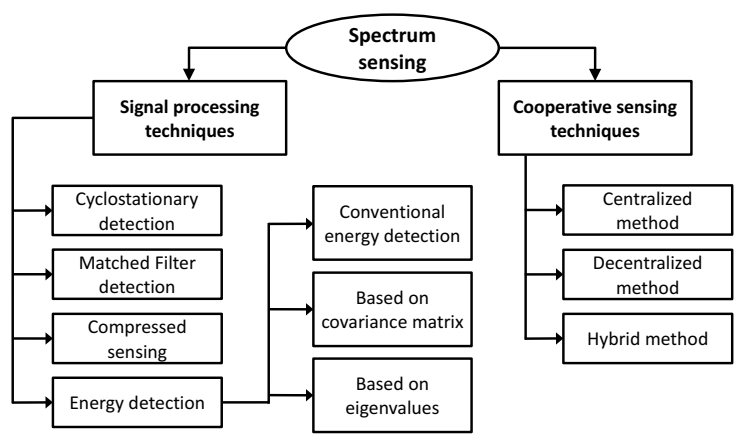

Fig. 1. Classification of spectrum sensing algorithms.

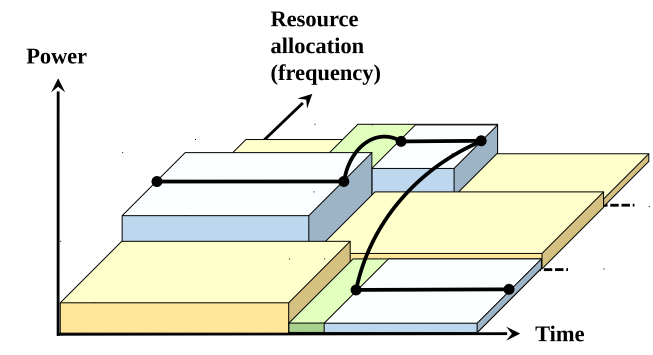

Resource used by PU

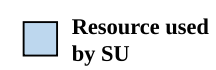

Free resource

Fig. 2. Scenario of dynamic resource allocation for secondary user over free frequency in narrowband context.

developed in the literature to ensure the dynamic resource allocation for the SU (Fig. 2). To improve the detection of the PU, some blind SS algorithms propose to advantageously exploit the multiple-input multiple-output (MIMO) configuration of some systems. In [7], the authors take advantage of the entries of the covariance matrix of the received signal. Two proposed methods are known to perform efficiently in very low SNR region: the first one is called the covariance absolute value (CAV) and the second one is called covariance Froebenius norm (CFN). Other recent contributions are based on the eigenvalue decomposition of the covariance matrix as maximum-to-minimum eigenvalue (MME) [8], arithmeticto-geometric mean (AGM) [9], blindly combiend energy detection (BCED) [10] and mean-to-square extreme eigenvalue (MSEE) [11]. Experimental evaluation points out the advan- 
tages of some sensing algorithms using the cyclostationarity of the signal [12]. The general likelihood ratio test (GLRT) is also one of the famous SS method that minimizes the sensing time and allows for a higher spectrum efficiency [13]. Other SS algorithms based on the energy and the beamforming are developed. Indeed, the maximum-to-minimum beam energy (MMBE) [14], maximum-to-mean energy detector (MMED) [15] and maximum energy beamforming-output-toinput (MEBOI) [16] detect only one PU under wave plane propagation assumption. In this paper, we propose a new algorithm based on the joint use of several SS methods using the covariance matrix and its eigenvalue decomposition. This proposed method, called unified spectrum algorithm (USA), takes advantage of the covariance matrix based algorithms firstly. In case where the PU is not detected, the eigenvalue decomposition is performed and all the SS algorithms are jointly processed to take the final decision.

The rest of the paper is organized as follows. In Section II, we present the background work including the system model and some SS algorithms based on the covariance matrix of the received signal and the eigenvalue decomposition. In Section III, we provide a detailed algorithm of the proposed method. In Section IV, the performance analysis is provided through simulations. Finally, in section V, our research contribution is concluded.

To describe vector and matrices, we respectively use boldface lower letter and boldface capital letter. We use superscript $(.)^{T}$ to denote transpose.

\section{SYSTEM MODEL AND BACKGROUND}

\section{A. System model}

We assume a SU terminal equipped with $M>1$ uniformly linear array antennas. We consider two main hypotheses $\mathcal{H}_{1}$, when the PU is present and $\mathcal{H}_{0}$, when the frequency band is free. The expression of the received signal samples under these two hypothesis are given by

$$
\begin{array}{ll}
\mathcal{H}_{0}: & y_{m}(n)=w_{m}(n) \\
\mathcal{H}_{1}: & y_{m}(n)=a_{m}(n)+w_{m}(n),
\end{array}
$$

where $a_{m}(n)$ represents the primary signals, $w_{m}(n)$ is a zeromean additive white Gaussian noise with variance $\sigma_{w}^{2}$ and $m$ represents the antenna $(m=0, \cdots,(M-1))$. The vector representation of the received signal, under $\mathcal{H}_{1}$ hypothesis, at the $\mathrm{SU}$ is given by

$$
\mathbf{y}(n)=\sum_{p=1}^{P} \sum_{k=0}^{C_{p}-1} \mathbf{h}_{p}(k) x_{p}(n-k)+\mathbf{w}(n),
$$

where $P$ is the number of $\mathrm{PU}\left(\mathrm{PU}_{p}, 1 \leqslant p \leqslant P\right)$ and $\mathbf{w}(n)=\left[w_{1}(n), w_{2}(n), \cdots, w_{M}(n)\right]^{T}$. We note $x_{p}$ the signal of the $p^{t h}$ PU. The channel order is denoted $C_{p}$ and $\mathbf{h}_{p}=$ $\left[h_{1 p}, h_{2 p}, \cdots, h_{M p}\right]^{T}$ is the expression of the channel from $\mathrm{PU}_{p}$ to $\mathrm{SU}$ and $C_{p}$ is the channel order. Thus the frequency selectivity is given by the order of the channel $C_{p}\left(C_{p}>0\right)$. The well-known smoothing factor $L$ [8] allows to represent consecutive samples, thus the expressions of the vectors are given by

$$
\begin{gathered}
\mathbf{y}_{L}(n)=\left[\mathbf{y}^{T}(n), \cdots, \mathbf{y}^{T}(n-L+1)\right]^{T}, \\
\mathbf{w}_{L}(n)=\left[\mathbf{w}^{T}(n), \cdots, \mathbf{w}^{T}(n-L+1)\right]^{T}, \\
\mathbf{x}_{L}(n)=\left[\mathbf{x}_{1}^{T}(n), \mathbf{x}_{2}^{T}(n), \cdots, \mathbf{x}_{P}^{T}(n)\right]^{T},
\end{gathered}
$$

where $\mathbf{x}_{p}(n)=\left[x_{p}(n), x_{p}(n-1), \cdots, x_{p}\left(n-L-C_{p}+1\right)\right]$. The matrix expression of the received signal is given by

$$
\mathbf{y}_{L}(n)=\mathbf{H x}_{L}(n)+\mathbf{w}_{L}(n),
$$

where $\mathbf{H}=\left[\mathbf{H}_{1}, \mathbf{H}_{2}, \cdots, \mathbf{H}_{P}\right]$ and $\mathbf{H}_{p}$ is a $M L \times\left(C_{p}+L\right)$ matrix which represents the channel between $p^{t h} \mathrm{PU}$ and the SU. The size of the channel matrix $\mathbf{H}$ is given by $M L \times$ $(C+P L)$ where $C=\sum_{p=1}^{P} C_{p}$. The covariance matrix of the received signal is defined as

$$
\mathbf{R}_{y}(n)=\mathbf{H R}_{x} \mathbf{H}^{H}+\sigma_{w}^{2} \mathbf{I}_{M L}
$$

where

$$
\begin{aligned}
\mathbf{R}_{y} & =E\left[\mathbf{y}_{L}(n) \mathbf{y}_{L}(n)^{H}\right] \\
\mathbf{R}_{x} & =E\left[\mathbf{x}_{L}(n) \mathbf{x}_{L}(n)^{H}\right] .
\end{aligned}
$$

The covariance matrix of the received signal is a Hermitian matrix, thus the eigenvalues, noted $\lambda_{i}$, are real numbers then we define $M L$ eigenvalues such as $\lambda_{1} \geqslant \lambda_{2} \geqslant \cdots \geqslant \lambda_{M L}$. The covariance matrix for a finite number of samples is given by

$$
\mathbf{R}_{y}(N)=\frac{1}{N} \sum_{k=1}^{N} \mathbf{y}_{L}(k) \mathbf{y}_{L}(k)^{H}
$$

where $N$ is the number of observed samples. The noise subspace size is equal to $(M-P) L-C$, so we can note $\lambda_{1}>\lambda_{2}>\cdots>\lambda_{C+P L}>\lambda_{C+P L+1}=\cdots=\lambda_{M L}=\sigma_{w}^{2}$.

\section{B. Spectrum sensing methods}

In this section we introduce some well-known SS algorithms based on the entries of the covariance matrix. The off-diagonal entries of the covariance matrix of the received signal, corresponds to the main difference, between $\mathcal{H}_{0}$ and $\mathcal{H}_{1}$, which is exploited by the two following algorithms.

a) Covariance Absolute Value (CAV): The statistical test, noted $T_{\mathrm{CAV}}$, of the CAV approach is based on the ratio between the sum of the absolute values of $\mathbf{R}_{y}$ by the sum of the absolute values of the diagonal entries of the covariance matrix [7], hence

$$
T_{\mathrm{CAV}}=\frac{\sum_{n=1}^{M L} \sum_{m=1}^{M L}\left|r_{n m}\right|}{\sum_{n=1}^{M L}\left|r_{n n}\right|} \underset{\mathcal{H}_{1}}{\stackrel{\mathcal{H}_{0}}{\lessgtr}} \gamma_{\mathrm{CAV}},
$$

where $r_{n m}$ represents all the elements of the estimated covariance matrix $\mathbf{R}_{y}$ and $\gamma_{C A V}$ is the threshold given by [7]

$$
\gamma_{\mathrm{CAV}}=\frac{1+(L-1) \sqrt{\frac{2}{N \pi}}}{1-Q^{-1}\left(P_{f a}\right) \sqrt{\frac{2}{N}}},
$$


where $Q^{-1}$ is the inverse Q-function which describes the tail distribution function of the standard normal distribution

$$
Q(t)=\frac{1}{\sqrt{2 \pi}} \int_{t}^{+\infty} \exp \left(-u^{2} / 2\right) \mathrm{d} u
$$

b) Covariance Frobenius Form (CFN): This approach proposes as statistical test the same comparison as CAV method by using Euclidean norm for each entries of $\mathbf{R}_{y}$

$$
T_{\mathrm{CFN}}=\frac{\sum_{n=1}^{M L} \sum_{m=1}^{M L}\left|r_{n m}\right|^{2}}{\sum_{n=1}^{M L}\left|r_{n n}\right|^{2}} \underset{\mathcal{H}_{1}}{\stackrel{\mathcal{H}_{0}}{\lessgtr}} \gamma_{\mathrm{CFN}} .
$$

The threshold $\gamma_{\text {CFN }}$ is approximated in [17] by

$$
\gamma_{\mathrm{CFN}} \geqslant \frac{1+N+L}{N+2-Q^{-1}\left(P_{f a}\right) \sqrt{8 N+40+\frac{48}{N}}} .
$$

In the sequel, we will present a non-exhaustive list of spectrum sensing algorithms based on the eigenvalue decomposition of the covariance matrix of the received signal $\mathbf{R}_{y}$.

c) Maximum-to-Minimum Eigenvalue (MME): The MME is a SS algorithm proposed in [8] and its statistical test is expressed as

$$
T_{\mathrm{MME}}=\frac{\lambda_{1}}{\lambda_{M L}} \underset{\mathcal{H}_{1}}{\stackrel{\mathcal{H}_{0}}{\lessgtr}} \gamma_{\mathrm{MME}},
$$

where $\gamma_{\text {MME }}$ is the threshold of the MME method. The analytic expression of the smallest and largest eigenvalues are $\lambda_{M L}=\frac{\sigma_{b}^{2}}{N}(\sqrt{N}-\sqrt{M L})^{2}$ and $\lambda_{1}=\frac{\sigma_{b}^{2}}{N}(\sqrt{N}+\sqrt{M L})^{2}$ respectively. The threshold $\gamma_{\text {MME }}$ is given by

$$
\gamma_{\mathrm{MME}}=\Delta\left(1+\phi F_{\mathrm{TW}}^{-1}\left(1-P_{f a}\right)\right),
$$

where $\Delta=\frac{(\sqrt{N}+\sqrt{M L})^{2}}{(\sqrt{N}-\sqrt{M L})^{2}}, \phi=\frac{(\sqrt{N}+\sqrt{M L})^{-2 / 3}}{(N M L)^{1 / 6}}$ and $F_{\mathrm{TW}}^{-1}$ is the inverse cumulative distribution function of the TracyWidom (TW) distribution.

d) Arithmetic to Geometric Mean (AGM): In [9], the authors propose a SS algorithm based on the ratio between the arithmetic mean and geometric mean of the eigenvalues. The statistical test is given by

$$
T_{\mathrm{AGM}}=\frac{\frac{1}{M L} \sum_{k=1}^{M L} \lambda_{k}}{\left(\prod_{k=1}^{M L} \lambda_{k}\right)^{1 / M L}} \underset{\mathcal{H}_{1}}{\lessgtr} \gamma_{\mathrm{AGM}},
$$

where $\gamma_{\mathrm{AGM}}$ is the threshold proposed in [18] by

$$
\gamma_{\mathrm{AGM}}=\sqrt{\frac{2}{M L N}} Q^{-1}\left(P_{f a}\right)+1 .
$$

e) Blindly Combined Energy Detection (BCED): This method is derived from the Energy Detector (ED) method. In SS, at least only one PU is necessary to decide hypothesis $\mathcal{H}_{1}$. Thus in [10], the following statistical test is proposed,

$$
T_{\mathrm{BCED}}=\frac{\lambda_{1}}{\frac{1}{M L} \sum_{k=1}^{M L} \lambda_{k}} \stackrel{\mathcal{H}_{0}}{\lessgtr} \gamma_{\mathrm{BCED}},
$$

where $\gamma_{\mathrm{BCED}}$ is the threshold analytically developed, in [19], considering the ratio of the largest eigenvalue divided by the trace of a Wishart matrix with $N$ degree of freedom. The asymptotic limit of the ratio follows a TW distribution but in order to get an accurate expression of the $P_{f a}$, the second derivative for the TW distribution must be added. Thus the closed-form expression of the threshold $\gamma_{\mathrm{BCED}}$ is quite complex to obtain. For this reason the expression of the $P_{f a}$ is sufficient

$$
\begin{aligned}
& P_{f a}=1-F_{\mathrm{TW}}\left(\frac{\gamma_{\mathrm{BCED}}-\mu_{N, M L}}{\sigma_{N, M L}}\right) \\
& +\frac{1}{M L N}\left(\frac{\mu_{N, M L}}{\sigma_{N, M L}}\right)^{2} F_{\mathrm{TW}}^{\prime \prime}\left(\frac{\gamma_{\mathrm{BCED}}-\mu_{N, M L}}{\sigma_{N, M L}}\right),
\end{aligned}
$$

where $F_{\mathrm{TW}}^{\prime \prime}$ represents the second derivate of $F_{\mathrm{TW}}$,

$$
\begin{aligned}
& \mu_{N, M L}=\left(1+\sqrt{\frac{M L}{N}}\right)^{2} \\
& \sigma_{N, M L}=N^{\frac{-2}{3}}\left(1+\sqrt{\frac{M L}{N}}\right)\left(1+\frac{1}{\sqrt{\frac{M L}{N}}}\right)^{1 / 3} .
\end{aligned}
$$

f) Maximum-Eigenvalue-to-the-Geometric-Mean (MEGM): MEGM algorithm is presented in [18], the authors propose as statistical test the ratio of the largest eigenvalue and the geometric mean of the eigenvalues

$$
T_{\text {MEGM }}=\frac{\lambda_{1}}{\left(\prod_{k=1}^{M L} \lambda_{k}\right)^{1 / M L}} \underset{\mathcal{H}_{1}}{\stackrel{\mathcal{H}_{0}}{\lessgtr}} \gamma_{\text {MEGM }} .
$$

The threshold is given by

$$
\gamma_{\mathrm{MEGM}}=\frac{F_{\mathrm{TW}}^{-1}\left(1-P_{f a}\right) \nu+\chi}{N},
$$

where $\nu=\sqrt{\chi}\left(\frac{1}{\sqrt{N-1}}+\frac{1}{\sqrt{M L}}\right)^{(1 / 3)}$ and $\chi=$ $(\sqrt{N-1}+\sqrt{M L})^{2}$.

g) Mean-to-Square Extreme Eigenvalue (MSEE):

In [11], the authors propose an algorithm similar to the AGM one using only the extreme eigenvalues. The statistical test is given by

$$
T_{\mathrm{MSEE}}=\frac{\lambda_{1}+\lambda_{M L}}{2 \sqrt{\lambda_{1} \lambda_{M L}}} \underset{\mathcal{H}_{1}}{\stackrel{\mathcal{H}_{0}}{\lessgtr}} \gamma_{\mathrm{MSEE}},
$$

where the threshold is expressed as

$$
\gamma_{\text {MSEE }}=G^{-1}\left(\Delta\left(1+\phi F_{T W}^{-1}\left(1-P_{f a}\right)\right)\right),
$$

and $G(x)=2 x^{2}-1+2 x \sqrt{x^{2}-1}$. 

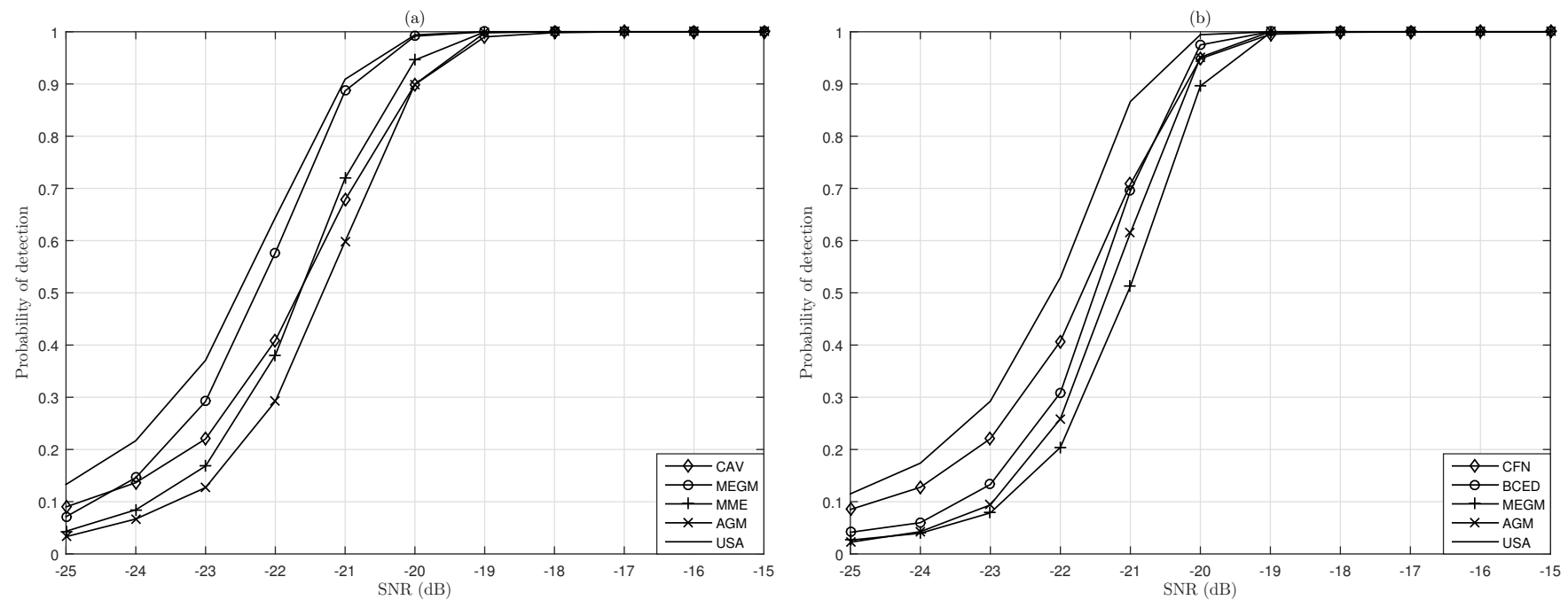

Fig. 3. Probability of detection versus SNR at different values of smoothing factor $L$. (a) $L=3$ and (b) $L=1$.

\section{UNIFIED SENSING APPROACH}

In this section, we introduce a new SS approach consisting in a cost effective unification of all detectors reminded in the previous section. The main idea of this new detector, called unified sensing algorithm (USA), is to improve the detection of the PU signal with less computational complexity. The decision of this new approach is based on two steps of detection. As a first step we only consider SS algorithms based on the covariance matrix and then, in the second step, we activate those based on the eigenvalue decomposition. Eigenvalue based detection are only performed when, in the first step, the algorithms based on the entries of the covariance matrix, do not detect any PU signal. The result of the comparison of each statistical test and its own threshold is a boolean expression denoted as $\mathcal{D}_{j}(j=\{\mathrm{CAV}, \mathrm{CFN}, \mathrm{MME}, \mathrm{AGM}$, BCED, MEGM, MSEE, USA $\}$ ). When $\mathcal{D}_{j}$ is true, the detector decides the presence of PU signal.

One of the objective of the SS, especially for noncooperative detectors, is to get the right decision on the occupancy of the spectral band as quickly as possible, i.e. relying on a number $N$ of samples the smallest as possible. Note that $N$, representing the sensing duration, is the value which defines the complexity of the sensing algorithm. However, to achieve the same performance, some method requires less sensing time depending on the detection parameters and the channel $\left(M, L, N\right.$ and $\left.C_{p}\right)$. In the presence of a PU $\left(\mathcal{H}_{1}\right)$ and in order to avoid eigenvalue decomposition, if the methods based on the entries of the covariance matrix of the received signal are sufficient then the USA method offers good performance with minimum complexity. In case where the eigenvalue-based methods are performed, then the proposal benefits from all the results based on the eigenvalue decomposition knowing that the thresholds are pre-computed from their analytical expression.

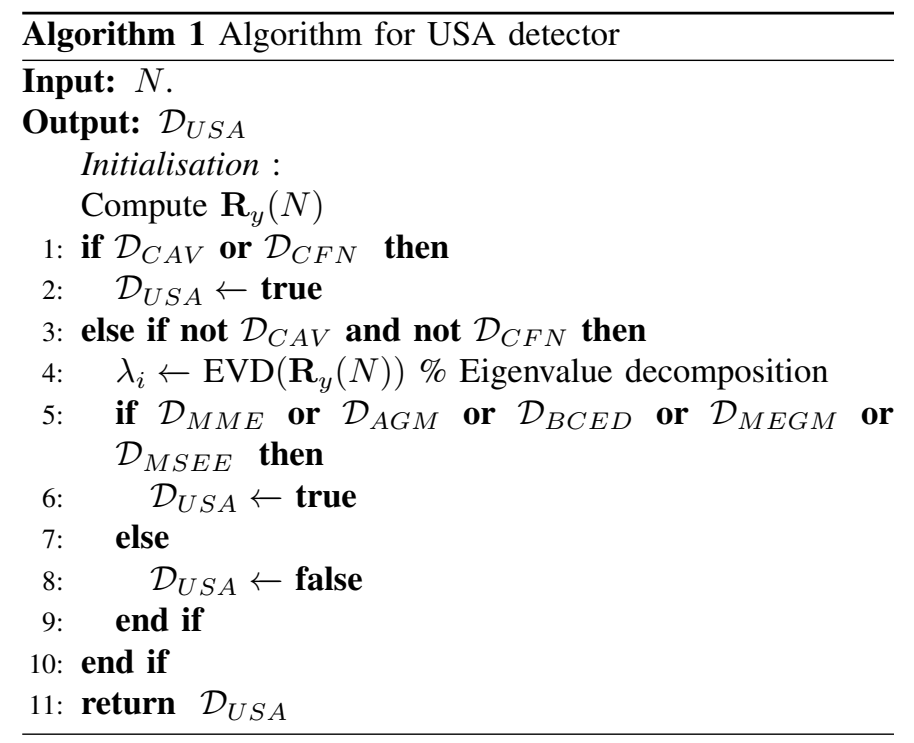

\section{Simulations AND Discussion}

In this section, we provide the simulation results in terms of the probability of detection versus the SNR for different features of detection. In the following simulations, we consider only one PU and the probability of false alarm is fixed at $P_{f a}=0.1$. For a sake of clarity, only some detection algorithms are drawn for different scenarios.

At first, Fig. 3 represents the detection performance at different values of the smoothing factor $L$. From this first figure, two interesting results can be observed. Firstly, the proposed USA algorithm provides the best results compared to all other detection methods, hereby confirming the positive effect of the joint approach. Indeed, the contribution of all the algorithms allow to improve the sensing significantly. Secondly, we note that the sensing methods based on the covariance matrix $(\mathrm{CAV}$ 

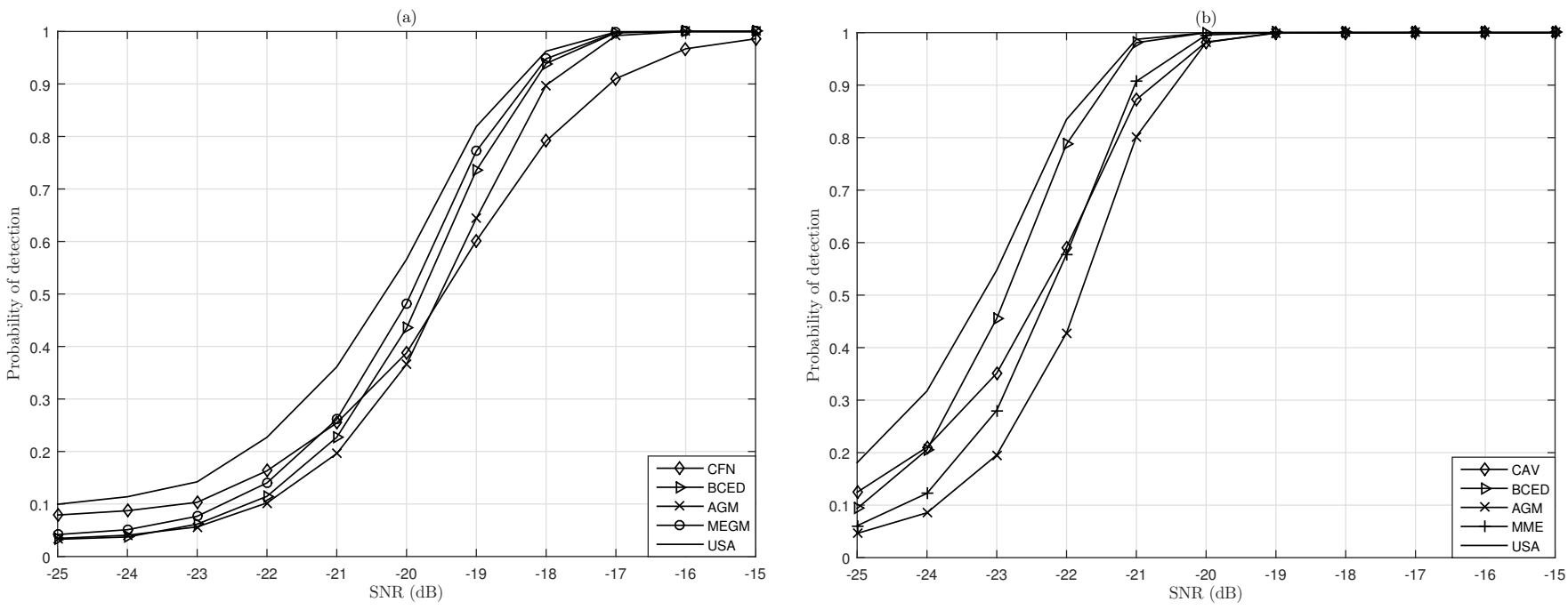

Fig. 4. Probability of detection versus SNR at different values of number of antenna $M$. (a) $M=4$ and (b) $M=10$.
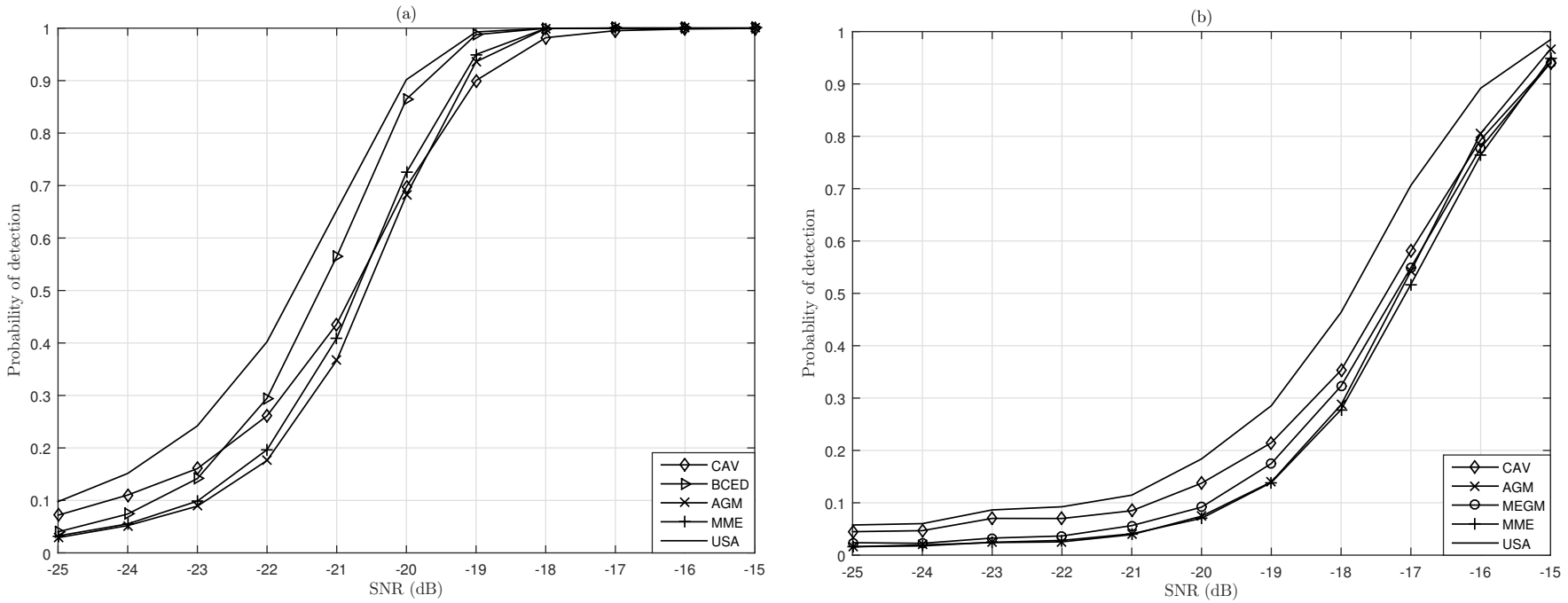

Fig. 5. Probability of detection versus SNR at different values of channel order $C_{p}$. (a) $C_{p}=0$ and (b) $C_{p}=5$.

and CFN) provide quite good performance at very low SNR region in both figure Fig. 3.a and 3.b. For example, at SNR $=-21 \mathrm{~dB}$, more than $70 \%$ of the right detection of the USA method is performed by the covariance matrix based algorithms.

In Fig. 4, we now illustrate the probability of detection versus SNR for different values of the number of antennas $M$. Comparing Fig. 4.a and 4.b, it appears that when the number of antennas is relatively low, methods based on the covariance matrix become much less accurate. For example, at $\mathrm{SNR}=-19 \mathrm{~dB}$, the contribution of $\mathrm{CAV}$ and CFN represent only $70 \%$ of the proposed algorithm in Fig. 4.a and approximately $100 \%$ in Fig. 4.b. It can hence be concluded that the robustness of the USA algorithm relies more on eigenvalue-based approaches (such as MEGM or MME) at small-scale antenna regime.

In Fig. 5 represents the probability of detection versus the SNR for different numbers of channel paths. It is observed that performances are very sensitive to multipath. However, the proposed method provides better detection, almost of $10 \%$ for example, for a SNR range from $-19 \mathrm{~dB}$ to $-16 \mathrm{~dB}$.

Finally, the impact of the sensing time $N$ on the performance is investigated in Fig. 6. We note that the gap of performance between all the methods remains globally the same whatever $N$. These results are expected because the influence of the detection time does not influence the performance differences between all the algorithms. Eventually, it clearly appears that that the the USA method offers better 

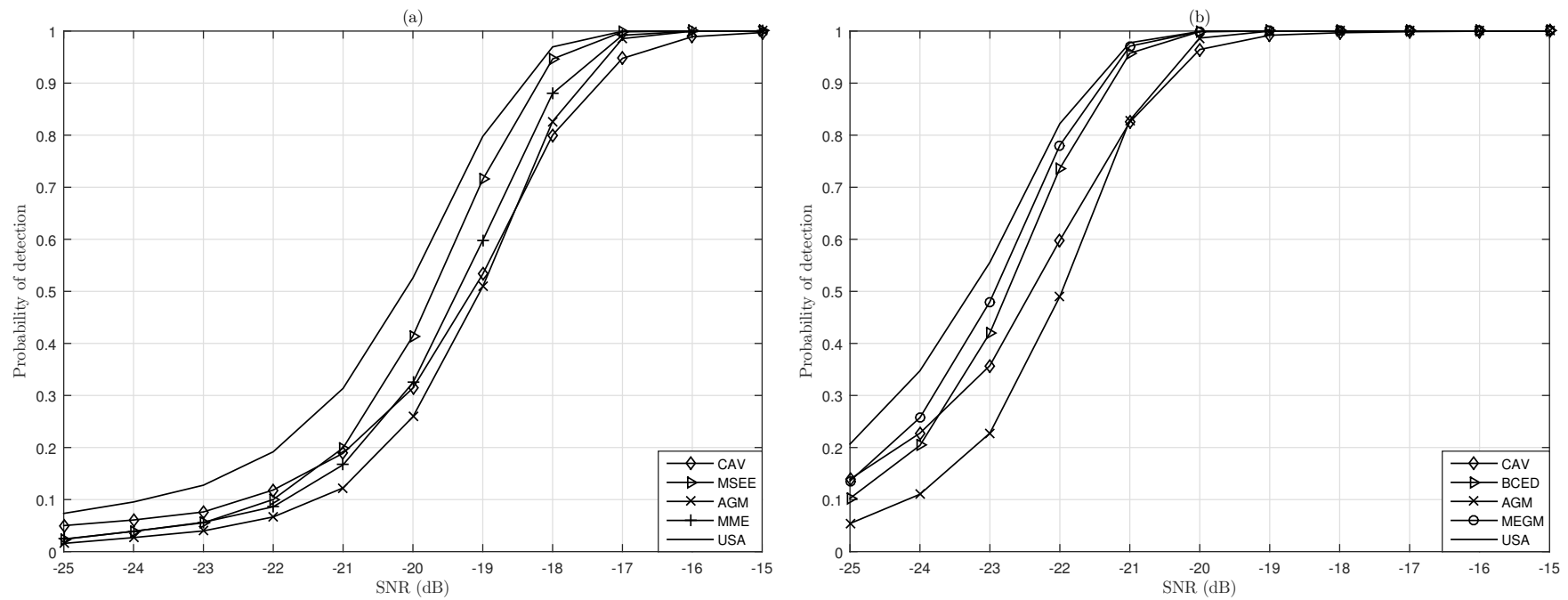

Fig. 6. Probability of detection versus SNR at different values of number of samples $N$. (a) $N=5000$ and (b) $N=20000$.

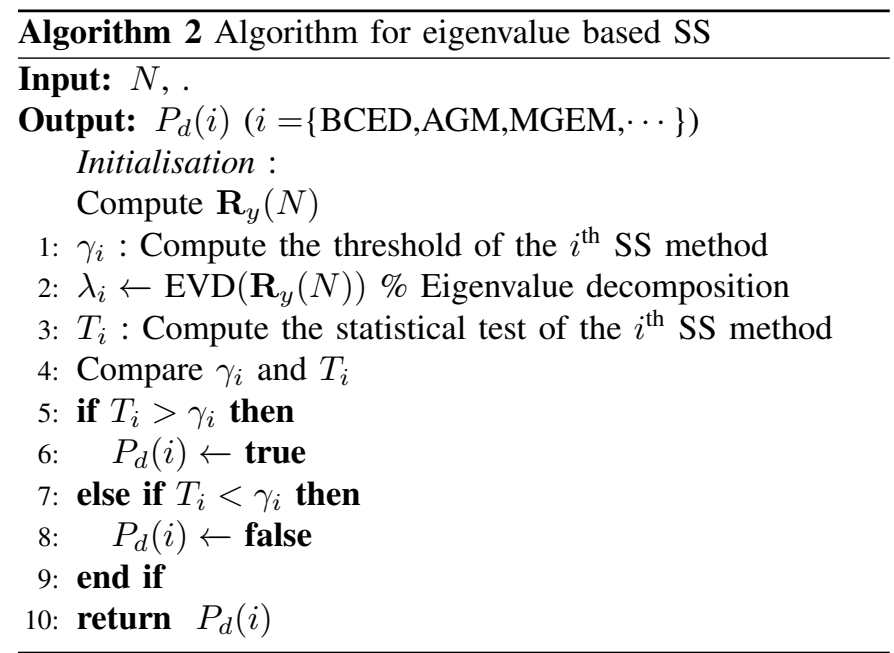

performance than the other methods.

\section{CONClusion}

In this paper, we proposed a new spectrum sensing algorithm which consists in the unification of miscellaneous sensing methods of the literature. Our joint algorithm, called unified spectrum algorithm (USA) is based on two steps of decision. First USA method exploits results from algorithms based on the covariance matrix of the received signal and then, in the case of miss-detection, these based on eigenvalue decomposition are activated. From simulation results and comparisons, we conclude that the proposed method outperforms the other ones for all the scenarios and system parameters such as the number of antennas, the smoothing factor, the multipath order of the channel or the sensing duration. This confirms that the proposed joint algorithm takes advantage of the different unitary sensing methods.

\section{REFERENCES}

[1] F. C. Comm., "Fcc," Facilitating Opportunities for Flexible, Efficient, and Reliable Spectrum Use Employing Cognitive Radio Technologies, Feb. 25, 2010

[2] J. Mitola and J. Maguire, G.Q., "Cognitive radio: making software radios more personal," IEEE Personal Communications, vol. 6, pp. 13-18, Aug. 1999.

[3] C.-W. Pyo, "Standard for ieee wran," 802.22 Working Group IEEE P802.22/D0.1 Draft Standard for Wireless Regional Area Networks, May 2006.

[4] M. Naraghi-Pour and T. Ikuma, "Autocorrelation-based spectrum sensing for cognitive radios," Vehicular Technology, IEEE Transactions on, vol. 59, pp. 718-733, Feb 2010.

[5] O. S. Mossad and M. ElNainay, "Genetic algorithm-based crowdsensing for cognitive radio networks," in 2018 14th International Conference on Wireless and Mobile Computing, Networking and Communications (WiMob), pp. 1-7, Oct 2018.

[6] K. Cichoń, A. Kliks, and H. Bogucka, "Energy-efficient cooperative spectrum sensing with a merged clustering measure," in 2017 IEEE 13th International Conference on Wireless and Mobile Computing, Networking and Communications (WiMob), pp. 38-43, Oct 2017.

[7] Y. Zeng and Y.-C. Liang, "Spectrum-sensing algorithms for cognitive radio based on statistical covariances," IEEE Transactions on Vehicular Technology, vol. 58, pp. 1804-1815, May 2009.

[8] Y. Zeng and Y.-C. Liang, "Eigenvalue-based spectrum sensing algorithms for cognitive radio," IEEE Transactions on Communications, vol. 57, pp. 1784-1793, Jun. 2009.

[9] R. Zhang, T. J. Lim, Y.-C. Liang, and Y. Zeng, "Multi-antenna based spectrum sensing for cognitive radios: A glrt approach," Communications, IEEE Transactions on, vol. 58, pp. 84-88, January 2010.

[10] Y. Zeng, Y. Liang, and R. Zhang, "Blindly combined energy detection for spectrum sensing in cognitive radio," IEEE Signal Processing Letters, vol. 15, pp. 649-652, 2008.

[11] K. Bouallegue, I. Dayoub, M. Gharbi, and K. Hassan, "Blind spectrum sensing using extreme eigenvalues for cognitive radio networks," IEEE Communications Letters, vol. 22, pp. 1386-1389, July 2018.

[12] A. Nafkha, B. Aziz, M. Naoues, and A. Kliks, "Cyclostationarity-based versus eigenvalues-based algorithms for spectrum sensing in cognitive radio systems: Experimental evaluation using gnu radio and usrp," in 2015 IEEE 11th International Conference on Wireless and Mobile Computing, Networking and Communications (WiMob), pp. 310-315, Oct 2015.

[13] A. Badawy and T. Khattab, "A hybrid spectrum sensing technique with multiple antenna based on glrt," in 2013 IEEE 9th International Conference on Wireless and Mobile Computing, Networking and Communications (WiMob), pp. 736-742, Oct 2013. 
[14] K. Bouallegue, I. Dayoub, M. Gharbi, and K. Hassan, "A cost-effective approach for spectrum sensing using beamforming," Physical Communication, vol. 22, no. Supplement C, pp. 1-8, 2017.

[15] K. Bouallegue, I. Dayoub, and M. Gharbi, "Spectrum sensing for wireless communications using energy ratio and beamforming," in 2017 IEEE International Conference on Communications (ICC), pp. 1-6, May 2017.

[16] K. Bouallegue, I. Dayoub, and M. Gharbi, "On low-complexity spectrum sensing: Analytical approach based spatial scanning," in 2017 IEEE Wireless Communications and Networking Conference (WCNC), pp. 16, March 2017.

[17] Y. Zeng and Y. Liang, "Covariance based signal detections for cognitive radio," in 2007 2nd IEEE International Symposium on New Frontiers in Dynamic Spectrum Access Networks, pp. 202-207, April 2007.

[18] N. Pillay and H. J. Xu, "Eigenvalue-based spectrum 'hole' detection for nakagami-m fading channels with gaussian and impulse noise," IET Communications, vol. 6, pp. 2054-2064, Sept 2012.

[19] B. Nadler, "On the distribution of the ratio of the largest eigenvalue to the trace of a wishart matrix," Journal of Multivariate Analysis, vol. 102, no. 2, pp. 363-371, 2011. 